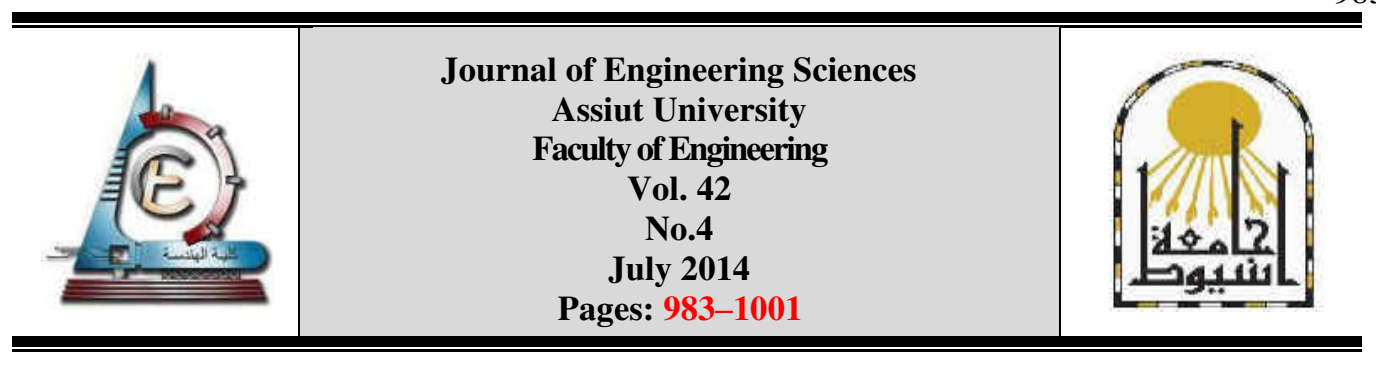

\title{
PROPOSED APPROACHES TO ENHANCE THE ORTHOGONAL FREQUENCY DIVISION MULTIPLEXING SYSTEM
}

\author{
Usama Sayed Mohammed* and Ahmed Tohamy \\ Communications \& Electronics Eng Dept., Faculty of Eng., Assiut University, Egypt \\ Recieved15 June 2014; Accepted 15 July 2014
}

\begin{abstract}
In the Orthogonal Frequency Division Multiplexing (OFDM) systems, where the data symbols are transmitted in parallel on different carriers, the length of a symbol is extended. This extension of the symbol length causes the OFDM system to be less sensitive to channel dispersion than a single carrier system transmitting data symbols at the same data rate. However, at the edges of the OFDM symbol, the channel dispersion still causes distortion, and hence introduces interference between successive symbols (i.e. Inter-Symbol Interference, ISI) and interference between different carriers within the same symbol (i.e. Inter-Carrier Interference, ICI). Different guard interval techniques for the OFDM transmission were suggested to reduce the interference between successive symbols. The most commonly used guard interval is the cyclic prefix (CP). In this paper, the impact of replacing the CP by zero insertion (ZI) before the Inverse Fast Fourier Transform (IFFT) process on the OFDM transmitter is studied. The motivation of using the ZI instead of the CP is the reduction in the transmission rate and the high performance in reducing the channel distortion. Moreover, the proposed ZI-OFDM system is suitable to minimize the effect of the fading channel when the channel characteristics are unknown or difficult to be estimated. Another approach to enhance the OFDM system is also introduced in this paper. A signal denoising approach is suggested to be added in the receiver to reduce the effect of the additive white Gaussian noise (AWGN) channel. In this approach, the Radio Frequency signal (RF) is enhanced using a wavelet thresholding technique instead of enhancing the baseband signal. The simulation results show that the proposed ZI approach provides better transmission performance than the recent techniques and achieves a $20 \%$ reduction in the data redundancy relative to the redundancy in the CP-OFDM system. Moreover, the results show that the proposed thresholding approach significantly removed the noise and the signal power is enhanced by almost $30 \mathrm{db}$ with different values of the channel signal-to-noise ratio (SNR). The overall system performance of the proposed ZI approach in the transmitter together with the thresholding approach in the receiver is tested in this paper.
\end{abstract}

Keywords: wavelet thresholding, Gaussian noise, signal denoising, Inter-Symbol Interference (ISI), Inter-Carrier Interference (ICI), cyclic prefix.

\footnotetext{
* Correspondence author.

Email address:usama@aun.edu.eg
} 
Usama S. Mohammed and Ahmed Tohamy, New approaches to enhance the orthogonal ............

\section{Introduction}

The Orthogonal Frequency Division Multiplexing (OFDM) is a special form of multicarrier modulation (MCM), where a single data stream is transmitted over a number of lower rate subcarriers. It is worth mentioning here that OFDM can be seen as either a modulation technique or a multiplexing technique. One of the main reasons to use OFDM is to increase the robustness against frequency selective fading and narrowband interference. In a single carrier system, a single fade or interferer can cause the entire link to fail, but in a multicarrier system, only a small percentage of subcarriers will be affected. Error correction coding can then be used to correct the few erroneous sub-carriers. In OFDM systems, where the data symbols are transmitted in parallel on $\mathrm{N}$ different carriers, the length T of a symbol is extended with a factor $N$ (J., 1990). This extension of the symbol length causes the OFDM system to be less sensitive to channel dispersion than a single carrier system transmitting data symbols at the same data rate. However, at the edges of the OFDM symbol, the channel dispersion still causes distortion, and hence introduces interference between successive symbols (ISI) and interference between different carriers within the same symbol (ICI). To reduce the effect of the ISI, each symbol is extended with a guard interval. When the length of the guard interval is longer than the duration of the channel impulse response, ISI can completely be removed. However, as the transmission efficiency reduces with the insertion of the guard interval (during the guard interval, no new information can be transmitted); the guard interval must be chosen sufficiently small. The most commonly used guard intervals are the cyclic prefix (Prasad, 1998), the Zero Padding (ZP), and the Known Symbol Padding (KSP) [14]. Fig.1 shows the CP-OFDM block diagram. The basic concept of the OFDM system and its applications is illustrated in many references(Nee \& Prasad, 2000; Noble, Sneddon, \& G. Eason, 1955).Cyclic Prefix is used in OFDM system to compensate (overcome) synchronization mismatch problem in a multipath fading channel, which are typically of significant order, because every echo component of the signal is poorly synchronized signal. As a consequence, the base pulses of the original OFDM signal and the delayed version of the signal are no longer orthogonal. This leads to several ISI in time and frequency as well because the detector output $\mathcal{D}_{\mathrm{kl}}\left[\mathrm{s}_{\tau}\right]$ at frequency number $\mathrm{k}$ and time slot $l$ of the delayed signal $\mathrm{s}_{\tau}(\mathrm{t})=\mathrm{s}(\mathrm{t}-\tau)$ with $0<\tau<T$ has ISI contributions from pulses at all subcarrier frequencies at time slot $l$ and $l-1$. The output of the detectorg ${ }_{\mathrm{k}^{\prime} 1^{\prime}}^{\prime}(\mathrm{t})$, given that the pulses $\mathrm{g}_{\mathrm{kl}}(\mathrm{t})$ has been transmitted is

$$
\left\langle g_{k l}, g_{k^{\prime} l^{\prime}}^{\prime}\right\rangle=\sqrt{\frac{T}{T_{S}}} \delta_{k k^{\prime}} \delta_{l l^{\prime}}
$$

The detector base pulses $\mathrm{g}_{\mathrm{k}^{\prime} \mathrm{l}^{\prime}}^{\prime} \mathrm{t}$ ) and the transmitted base pulses $\mathrm{g}_{\mathrm{kl}}(\mathrm{t})$ are orthogonal unless both time and the frequency index are identical. It is important to note that if they are identical the output does not take the value 1 but the smaller value $\sqrt{\mathrm{T} / \mathrm{T}_{\mathrm{S}}}$. This can be understood as a waste of energy by transmitting a part of the symbol (guard interval) that is not used for detection. The OFDM signal has the advantage of averaging fades when its symbol duration is longer than the length of the fades. Due to this fact, the OFDM symbols are partially destroyed under fading. This can be achieved by adopting a large number of subcarriers but at the cost of increasing the system complexity and resulting in a poor peak-to-average power (PAPR) ratio [3]. The correlation between the samples during one 
OFDM symbol must be taken into consideration. A comparison among the cyclic prefix, zero padding, and known symbol padding systems is shown in [5].

Another distortion in the OFDM signal is often due to the effect of the additive white Gaussian noise (AWGN) channel. To reduce the effect of the AWGN channel, a signal denoising approach is suggested in the receiver. In this approach, the radio frequency signal (RF) is enhanced using a wavelet thresholding technique instead of the baseband signal.

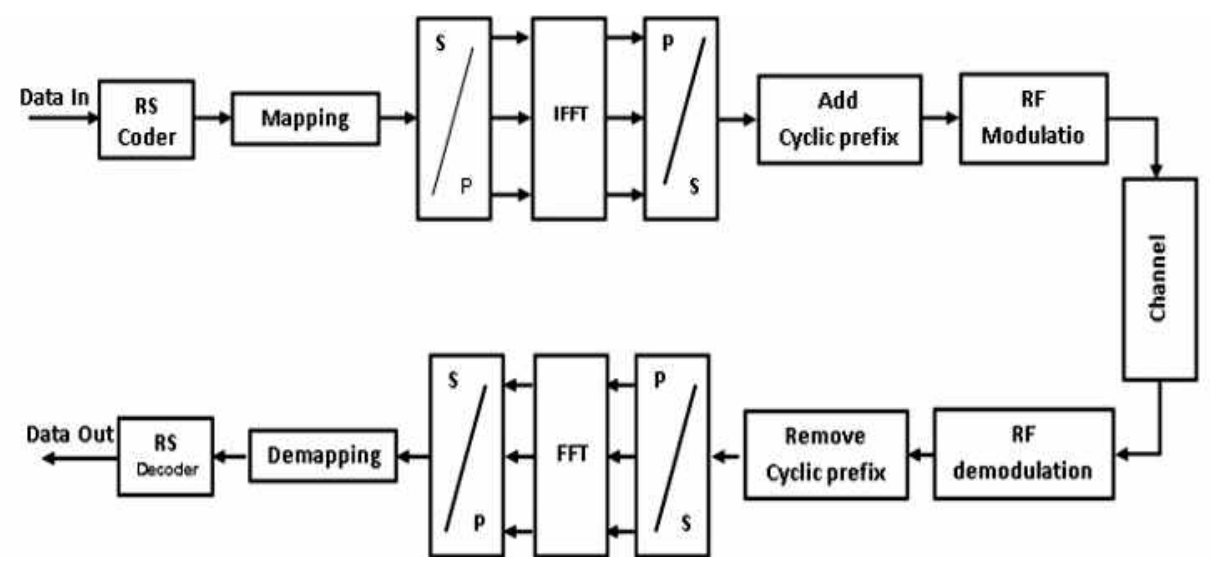

Fig.1. Original OFDM System with $\mathrm{CP}$ as a guard interval

The paper is organized as follows: Section 2 introduces system model and the performance analysis. Performance evaluation of the proposed system is given in section 3 . Finally the conclusions are made in section 4.

\section{System analysis for the proposed modified OFDM system}

A new approach to enhance the performance of the OFDM system, when the channel characteristics are unknown or difficult to be estimated, is introduced.

In this paper, the codes have been written for data transmission using an OFDM technique over two types of channels. The effect of fading channel and additive white Gaussian noise (AWGN) channel are studied and suggested approaches are used to enhance the performance of the OFDM system. In this work, the impact of replacing the Cyclic Prefix (CP) by zero insertion (ZI) before the IFFT process in the OFDM is studied. Associated with each OFDM symbol, the zeros will be added in the transmitter before the IFFT process. For symmetry of the OFDM symbol, the zeros will be inserted with the same length among the OFDM frames. The ZI acts as a safe region of the high frequency component (details) of the signal where delayed information from the previous symbols freely corrupts the low frequency components, and the high frequency components are protected by giving it a high immunity (higher SNR) against the additive noise. The motivation of using the ZI instead of the cyclic prefix is the reduction in the transmission rate and the high performance in reducing of the channel distortion. The performance comparison among the proposed ZI technique, the $\mathrm{CP}$, the Zero Padding (ZP), and the Known Symbol Padding (KSP) will be introduced in the simulation results. Another type of channels is the AWGN channel. The AWGN is a noise that affects the transmitted 
signal when it passes through the channel. It contains a uniform continuous frequency spectrum over a particular frequency band. The OFDM system has been built to reduce the effect of fading channel without the enhancement of the effect of the AWGN channel. In this work the approach of the wavelet thresholding denoising at the beginning of the receiver is used to denoise the received radio frequency (RF) signal.Fig.2 shows the proposed ZI-OFDM system.

The transmitted and the received signals for CP-OFDM, ZP-OFDM [14], KSP-OFDM, and ZI-OFDM are shown in Fig.3 and Fig.4; respectively.

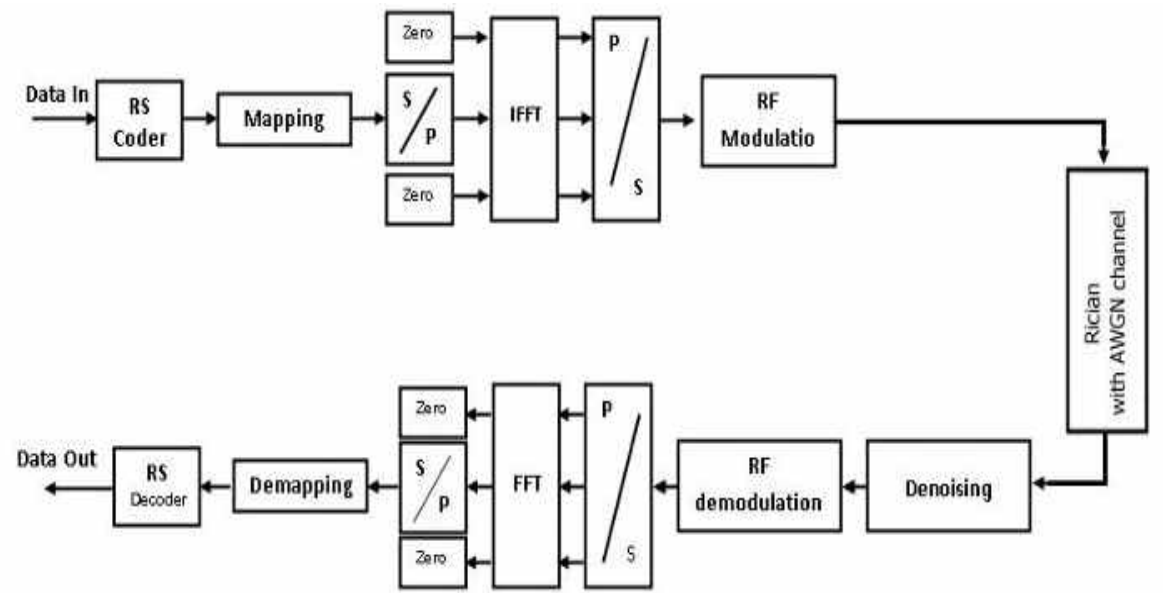

Fig. 2. Modified OFDM system
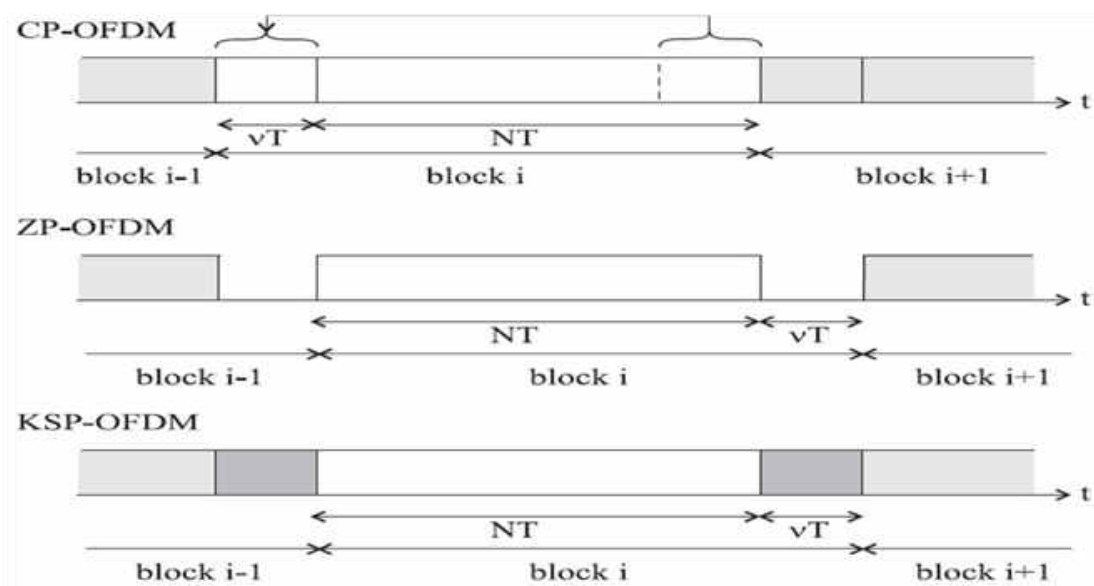

ZI-OFDM

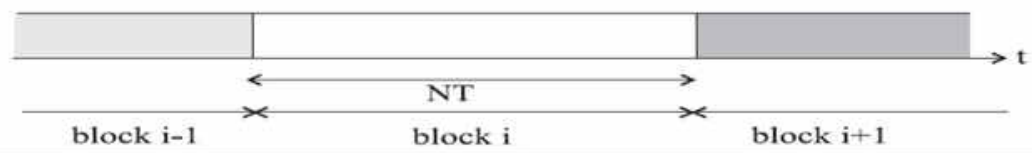

Fig. 3. Transmitted signal for CP-OFDM, ZP-OFDM, KSP-OFDM, and ZI-OFDM 
JES, Assiut University, Faculty of Engineering, Vol. 42, No. 4, July 2014, pp. 983-1001

The proposed Modified OFDM system can be described as follows:

1. The input signal is protected using Reed-Solomon (RS) encoder, and converting its output data to binary data stream.

2. Mapping the binary stream using Quadrature Phase Shift Keying (QPSK).

3. Passing the mapped stream through serial to parallel converter to fit the number of non-silence subcarrier (1536) (as the DAB model I standard).

4. Covering each frame with 256 zeroes at both ends.

5. The Inverse Fast Fourier Transform (IFFT) for each data frame ( $\mathrm{N}=2048$ bits; DAB model I ) is applied (Bracewell, 2000).

6. Passing the data through a parallel to serial converter.

7. Modulating of the output data using the RF modulator is achieved.

8. In the first stage of the receiver the wavelet thresholding system is used to denoise the received radio frequency $(\mathrm{RF})$ signal.
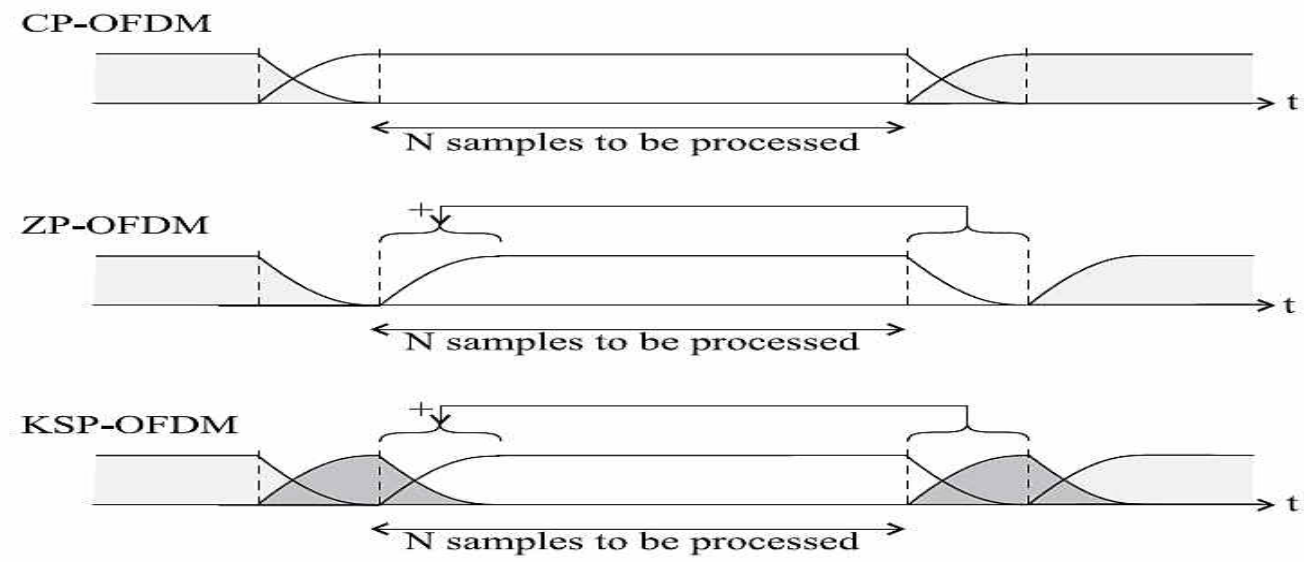

\section{ZI-OFDM}

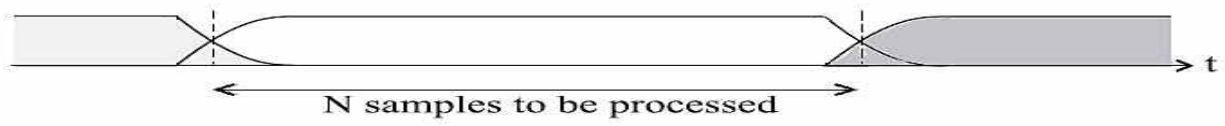

Fig. 4. Received signal for CP-OFDM, ZP-OFDM, KSP-OFDM, and ZI-OFDM

In the proposed ZI-OFDM system, data symbols $\mathrm{a}_{\mathrm{i}}$ are applied to zero padding before the IFFT processing. Then, the inverse FFT is applied resulting in the time-domain samples as follows:

$$
s_{i, Z I}=F^{+}\left(\Psi\left\|a_{i}\right\| \Psi\right)
$$

Where the $(1) \times\left(\frac{1}{2} v\right)$ matrix $\Psi=\operatorname{zeros}\left(1, \frac{1}{2} v\right)$ is the zero-padding operator, $A \| B$ is the concatenation operation between $\mathrm{A}$ and $\mathrm{B}$, and $\mathrm{s}_{\mathrm{i}, \mathrm{ZI}}=\left\{\mathrm{s}_{\mathrm{i}, \mathrm{ZI}}(\mathrm{k}) \mid \mathrm{k}=0, \ldots, \mathrm{N}-1\right\}$. At the receiver, the whole $\mathrm{N}$ samples are applied to the FFT, and then the zeros are removed from the first $\frac{1}{2} v$ samples, and the last $\frac{1}{2} v$ samples from the data part. The $\mathrm{N}$ outputs $\mathcal{Y}_{\mathrm{ZI}}(\mathrm{n})$ of the FFT can be written as follows: 


$$
y_{Z I}=\sum_{i=-\infty}^{+\infty} F H^{(i)} s_{i, Z I}+F \Lambda w
$$

The $\mathrm{n}^{\text {th }}$ FFT output can be rewritten as

$$
\mathcal{Y}_{Z I}(n)=\sum_{i=-\infty}^{+\infty} \sum_{n^{\prime}=0}^{N-1} a_{i}\left(n^{\prime}\right) \gamma_{i, Z I}\left(n, n^{\prime}\right)+W(n)
$$

Where

$$
\gamma_{i, Z I}\left(n, n^{\prime}\right)=\frac{1}{N} \sum_{k=0}^{N-1} \sum_{k^{\prime}=0}^{N-v} h\left(k-k^{\prime}-i(N) ; k\right) e^{-j 2 \pi \frac{k n-k^{\prime} n^{\prime}}{N}}
$$

Using a similar analysis as for CP-OFDM, it can be shown that the SINR at the FFT outputs for ZI-OFDM is independent of the carrier index $n$ and yields

$$
\operatorname{SINR}=\frac{E_{S} P_{U}}{E_{S} P_{I}+P_{N}}
$$

Neglecting the effect of the noise

$$
\operatorname{SINR}=\frac{P_{U}}{P_{I}}
$$

Then, the noise component $\mathrm{P}_{\mathrm{N}}=\mathrm{N}_{0}$, i.e. the value of the noise power is the same as in the case of the CP-OFDM. Taking into account the effect of the power efficiency loss in the CP-OFDM, it supports our clime that the ZI-OFDM system is better than the CPOFDM system.

\section{Performance evaluation of the proposed system}

\subsection{Performance analysis of the proposed approach with the Rician Fading channel:}

Matlab will be our simulator in the performance test, path gain is varied from -0.26 to 0 $\mathrm{dBs}$. However, the path delay is varied form $10^{-9}$ to $10^{-5}$ second for the same path. Fig.5 shows the mean square error (MSE) of the output signal when the channel gain was -0.16 $\mathrm{dB}$ with different path delays when the input signal is a random data. Fig.6 shows the same result for the same input data but when the path gain was $-0.06 \mathrm{~dB}$, and Fig.7 shows the relation between the averages of the MSE for the path delay against the path gain for random input signal. Fig. 8 and Fig.9 show the same previous relations when the input is a speech signal. However, Fig.10 shows the relation between the averages of the MSE for the path delay against the path gain for the speech input signal. For the image data input, the same tests are done and the results are shown in Fig.11 to Fig.13. From these results, it is clear that the performance of the proposed system outperforms the original CP-OFDM system in most cases. It is clear that, for input image data, the ratio between the average of the MSE in the case of the proposed system and the average of the MSE in the case of the CP-OFDM system is 1.8231. Fig.14 shows the perceptual quality of the transmitted image (monalisa) and the received image using the CP-OFDM system and the proposed system when the path gain is $-0.26 \mathrm{~dB}$ and the path delay is $10^{-5}$ second. However, Fig. 15 shows the transmitted speech signal and the received speech signal from the CP-OFDM system and the proposed system when the path gain is $-0.26 \mathrm{~dB}$ and the path delay is $10^{-5}$ second. 


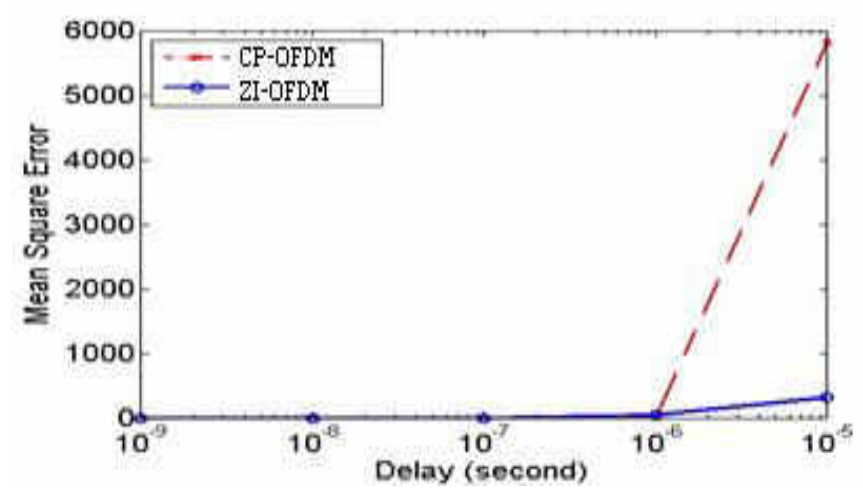

Fig. 5. MSE versus path delay in seconds, at path gain $=-0.16 \mathrm{~dB}$ for random input data

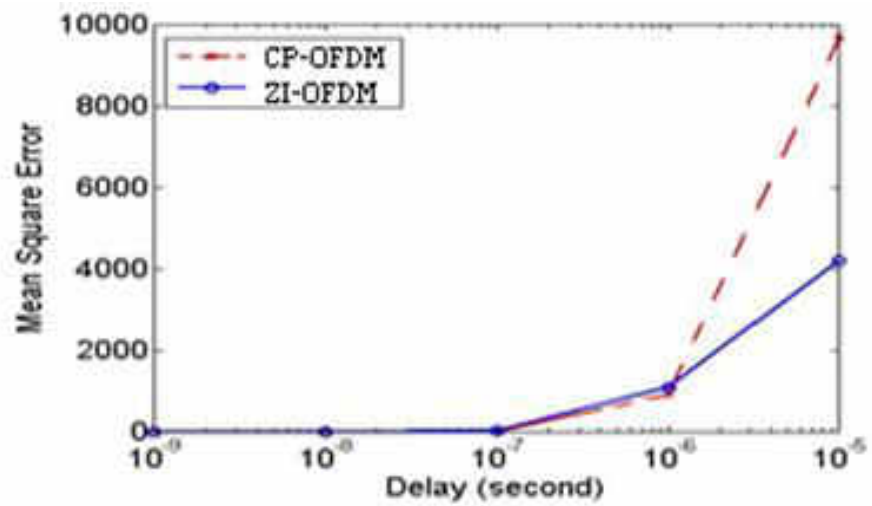

Fig. 6. MSE versus path delay in seconds, at path gain $=-0.06 \mathrm{~dB}$ for random input data

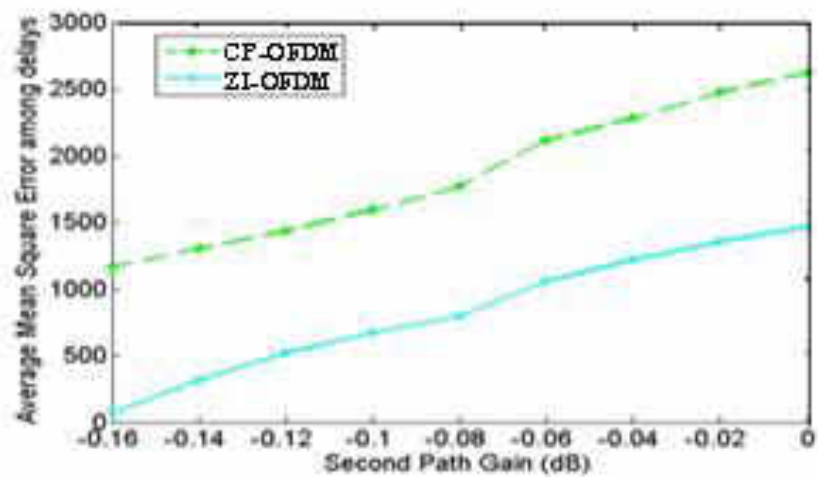

Fig. 7. Average MSE versus path gain in $\mathrm{dB}$, for random input data 


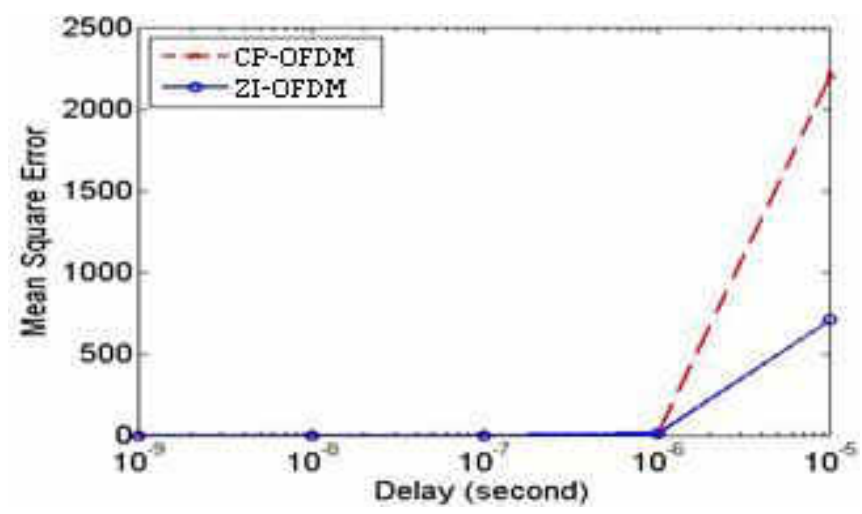

Fig. 8. MSE versus path delay in seconds, at path gain $=-0.16 \mathrm{~dB}$ for speech input data

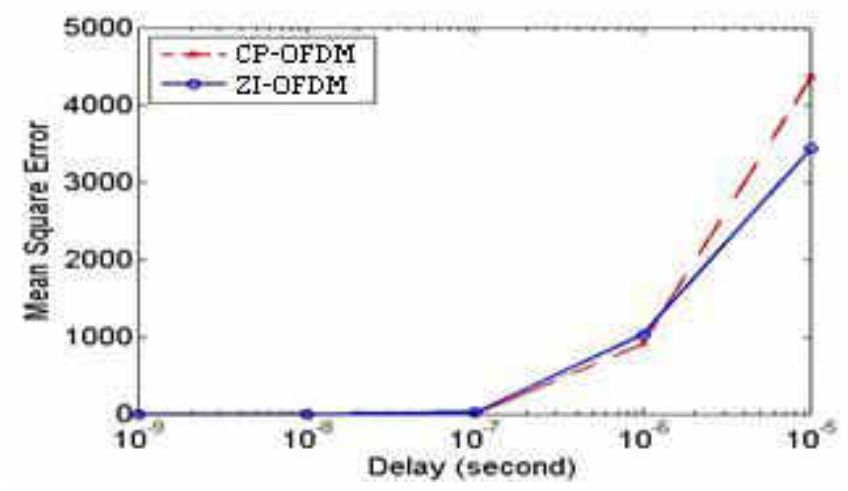

Fig. 9. MSE versus path delay in seconds, at path gain $=-0.06 \mathrm{~dB}$ for speech input data

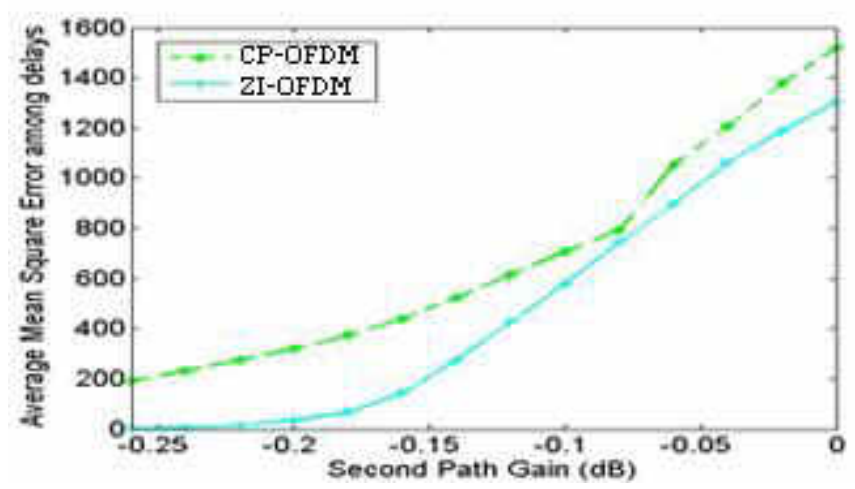

Fig. 10. Average MSE versus path gain in $d B$, for speech input data 


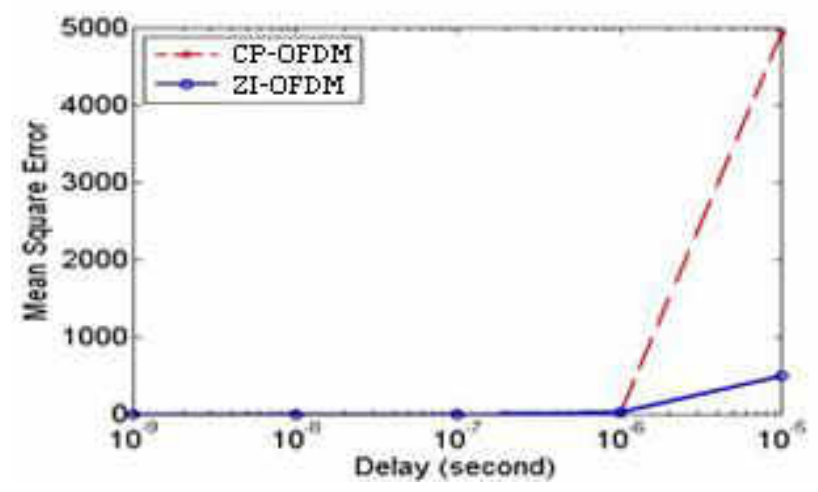

Fig. 11. MSE versus path delay in seconds, at path gain $=-0.16 \mathrm{~dB}$ for image input data

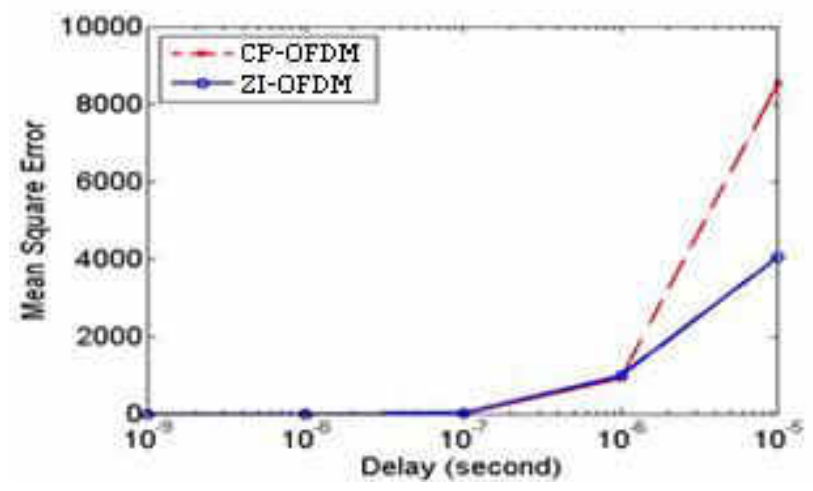

Fig. 12. MSE versus path delay in seconds, at path gain $=-0.06 \mathrm{~dB}$ for image input data

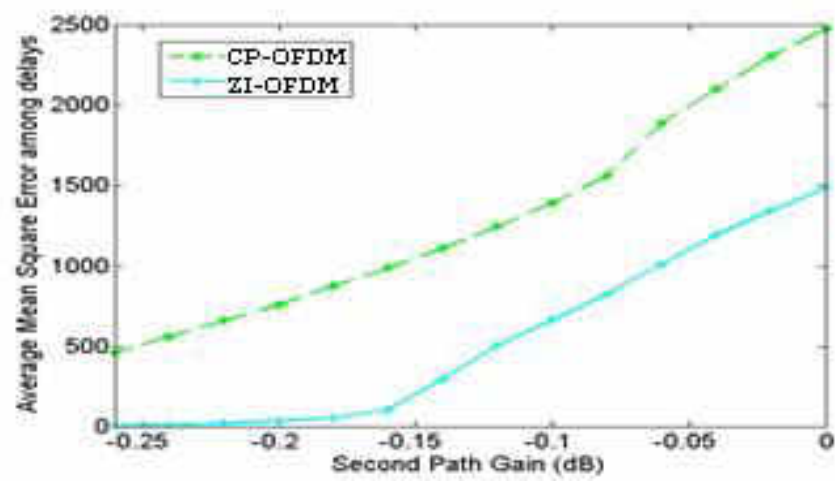

Fig. 13. Average Mean Square Error versus path gain in $\mathrm{dB}$, for image input data 


\section{Source Image}

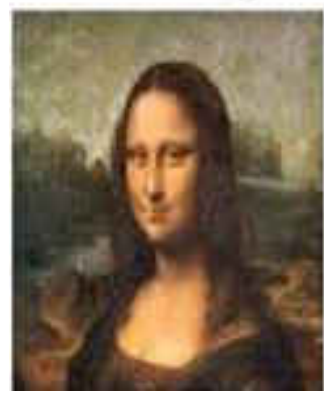

CP-OFDM

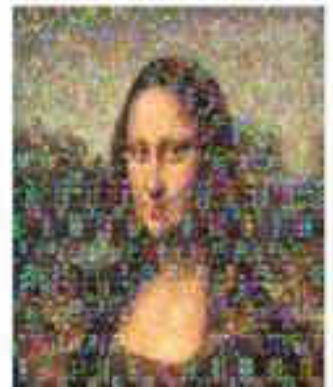

ZI-OFDM

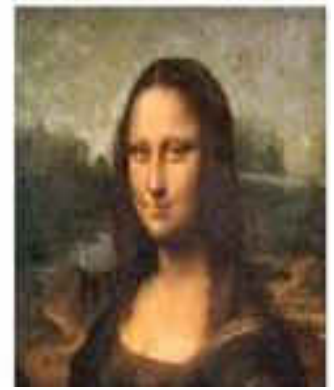

Fig. 14.Monalisa Image as a source and received images from CP-OFDM and ZIOFDM systems at path gain $=-0.26 \mathrm{~dB}$ and delay $=\mathbf{1 0}^{-\mathbf{5}}$

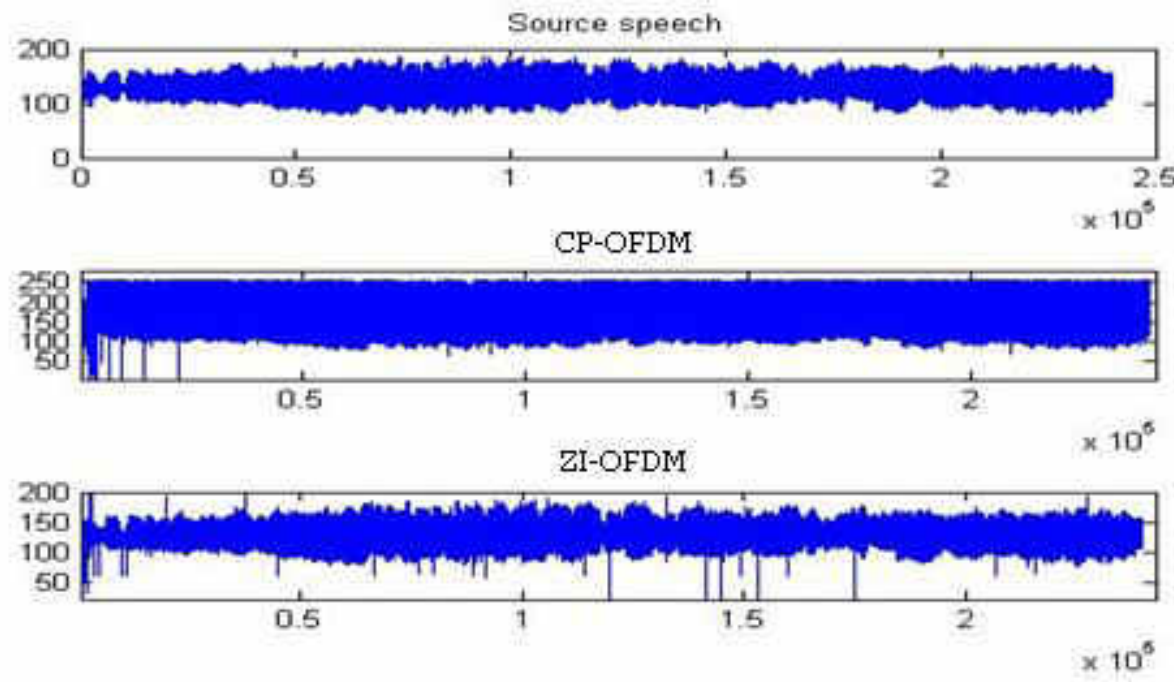

Fig. 15.TransmittedSpeech signal and received speech signal from the $\mathrm{CP}$ OFDM and ZI-OFDM system at path gain $=-0.26 \mathrm{~dB}$ and delay $=\mathbf{1 0}^{-\mathbf{5}}$

3.2. Performance analysis of the proposed approach with the AWGN channel only:

In general, the OFDM system is not proposed to deal with this type of channel, so our proposed denoising technique work with the OFDM systems and give a good result in the AWGN environment. With the birth of the wavelet theory and multi-resolution analysis, a signal denoising technique based on the wavelet transform has been extensively studied and tremendously improved. The denoising and feature detection of signals using the wavelet transform is done by representing the signal by a small number of coefficients. This wavelet shrinkage is based on thresholding, as developed by Donoho and Johnstone (Donoho \& Johnstone, 1994). The signal is composed into L levels before thresholding is applied. Fig.16 demonstrates the two types of thresholding, hard and soft thresholding, with threshold $\delta$.Hard thresholding zeroes out small coefficients, resulting in an efficient representation. 

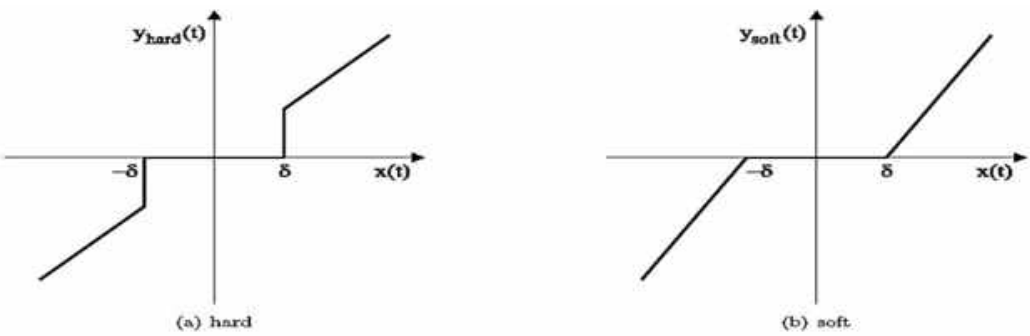

Fig. 16. Thresholding technique

Soft Thresholding softens the coefficients exceeding the threshold by lowering them using the threshold value. Soft thresholding gives better compression performance. When the thresholding value is applied, no perfect reconstruction of the original signal is possible. The outputs of soft and hard thresholding can be written as

$$
\begin{gathered}
y_{\text {hard }}= \begin{cases}\mathrm{x}(\mathrm{t}), & |\mathrm{x}(\mathrm{t})|>\delta \\
0, & |\mathrm{x}(\mathrm{t})| \leq \delta\end{cases} \\
y_{\text {soft }}= \begin{cases}\operatorname{sign}(\mathrm{x}(\mathrm{t}))(|\mathrm{x}(\mathrm{t})|-\delta), & |\mathrm{x}(\mathrm{t})|>\delta \\
0, & |\mathrm{x}(\mathrm{t})| \leq \delta\end{cases}
\end{gathered}
$$

The denoising is not limited to a special kind of noise; different kinds of disturbances can be filtered out of the images. Thresholding generally gives a low-pass version of the original signal. An appropriate threshold $\delta$ (the noise variance in the wavelet domain is used in this work) can suppress noise present in a signal. For denoising applications, generally soft thresholding is used. It is assumed that the noise power is smaller than the signal power. If this is not the case, the denoising by thresholding removes, besides the noise, a large part of the signal or leaves a larger part of the noise in the signal. In general, removing noise from the original signal is still a challenging problem for researchers. Most of the denoising techniques work at the baseband signals; they use image denoising as a major field to apply them researches (Kaur, Gupta, \& R.C. Chauhan, 2012). Adaptive (reconfigurable) (Latha \& M., 2011)(Liu, Teng, \& Chen, 2010), nonlinear (Gao, Sultan, $\mathrm{Hu}$, \& Wen-wen, 2010), and fixed thresholding (Kim \& Barner, 2005) are the most common techniques to perform the denoising at this band. In the OFDM system, the wavelet OFDM (OFDM-DWT) (M \& Dr.B.V.Uma, 2013)gives an OFDM system with high immunity against the AWGN channel and performs the denoising for OFDM signals. The following simulation results prove that the proposed system outperform the performance of the OFDM-DWT.

To select the best mother wavelet function, the RF modulated OFDM signal is processed by some wavelet functions to select the best denoising technique.

Different mothers wavelet are used to test the RF modulated signal. The RF modulated OFDM signal for color image is analyzed using various mothers wavelet functions as Daubechies (db5), Symlets (sym2), Coiflets (coif1), Biorsplines (bior2.2), reversebior (rbio4.4), DMeyer (dmey), and Haar (haar) functions; as an example in Fig.17 to Fig. 20. In these figures, $s$ is the signal, $d$ is the detail component, and $a$ is the approximate component; the sub-scripted number is the decomposition level of the wavelet transform. 
994

Usama S. Mohammed and Ahmed Tohamy, New approaches to enhance the orthogonal

For noise free signal, the simulation results prove that both the approximate and the detail coefficients at level 3 have zero value when the Haar wavelet function is used as shown in Fig.20 and Fig. 21. We exploit this feature and propose the denoising technique for the OFDM signal. The proposed algorithm is based on soft and hard thresholding. Fig.21 shows the analysis of the same RF modulated OFDM signal using the Haar wavelet at level 3 but after adding AWGN. It's clearly that we have information about the noise at the approximate and detail coefficients in level 3. Both the approximate and detail components in level 3 can be combined together to create the approximate component of level 2. So all the information can be gotten from this component will describe the noise characteristics. This characteristic (noise standard deviation $\sigma$ ) is used to perform the signal denoising process.

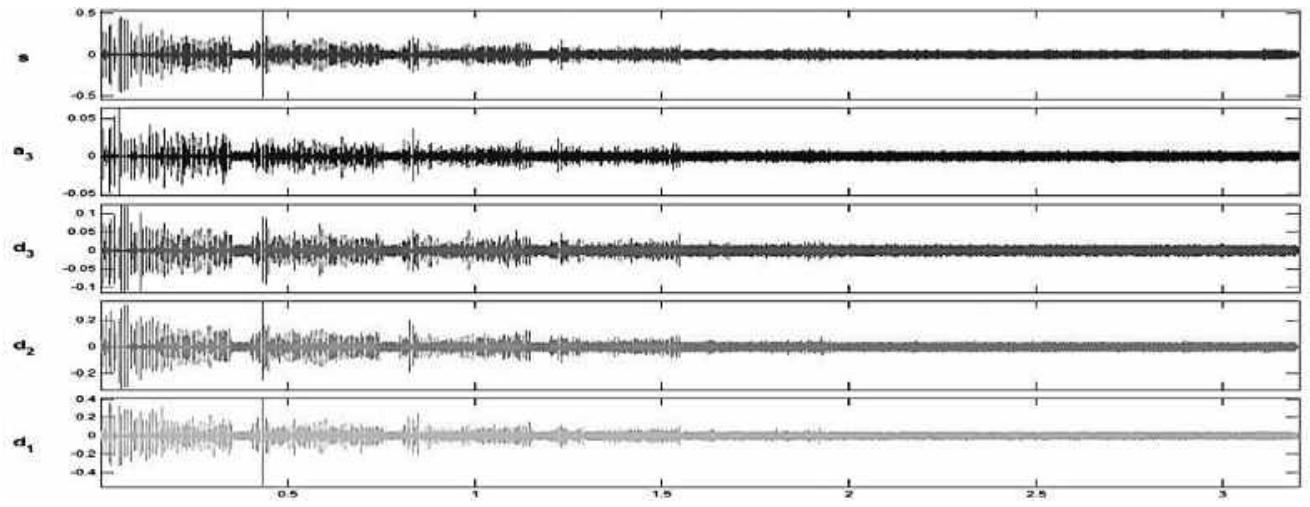

Fig. 17. OFDM signal level 3 decomposition using Daubechies (db5) wavelet

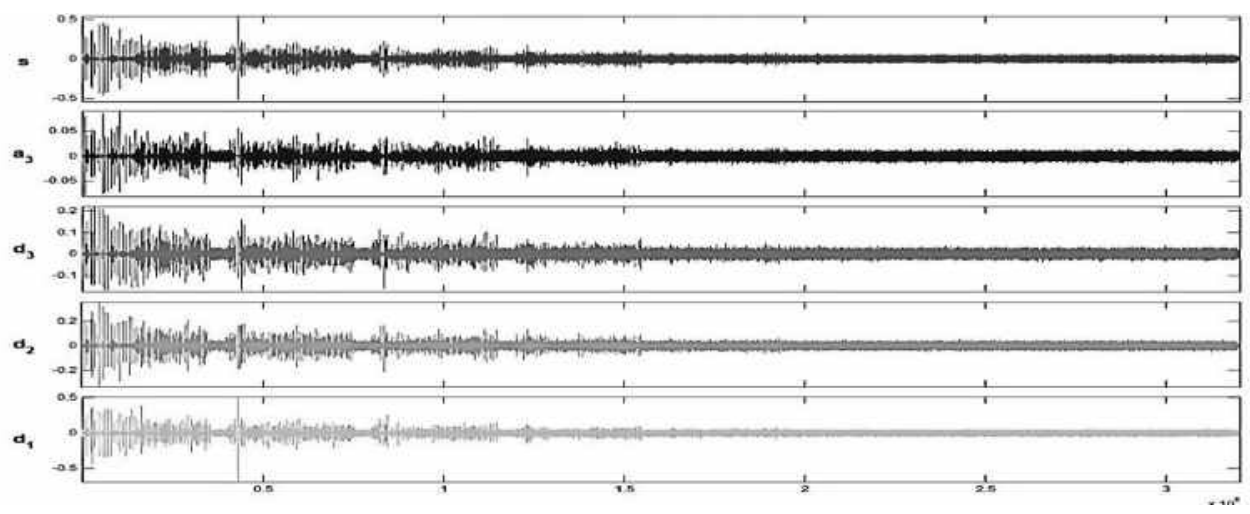

Fig. 18. OFDM signal level 3 decomposition using Symlets (sym2) wavelet 


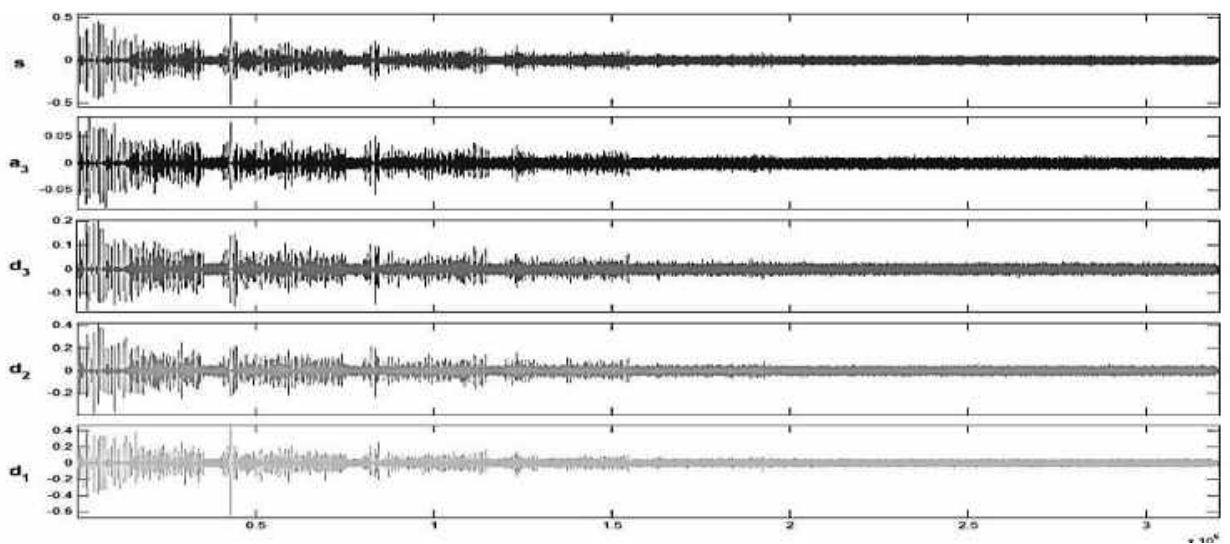

Fig. 19. OFDM signal level 3 decomposition using Coiflets (coif1) wavelet

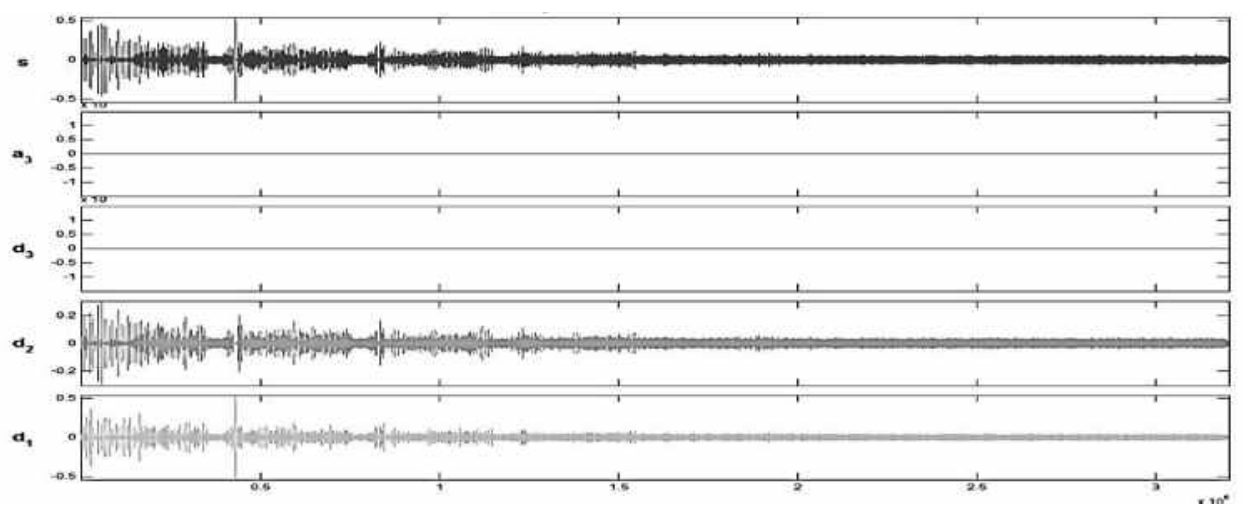

Fig. 20. OFDM signal level 3 decomposition using Haar (haar) wavelet

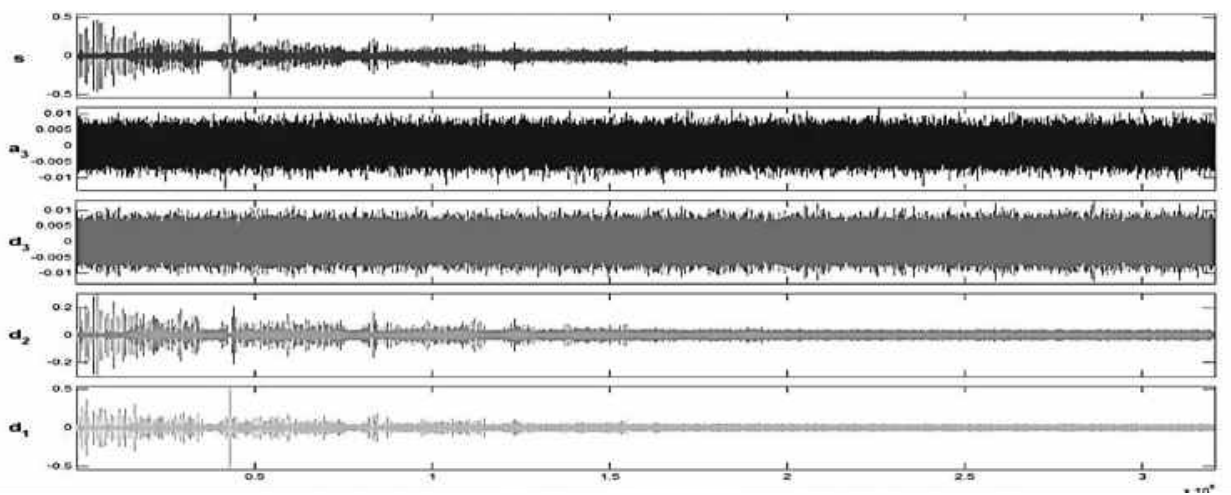

Fig. 21. Noisy OFDM signal with level 3 decomposition using Haar wavelet

The steps of the proposed technique in the wavelet domain after using the Haar wavelet transform are described as follows:

1. The noise standard deviation $\sigma_{2}$ will be estimated from the approximate coefficients in level 2.

2. Using $\sigma_{2}$ to perform the signal denoising process for the detail components in level 2 . 
3. Set the approximate coefficients at level 2 to zero.

4. Combine level 2 coefficients together to get the denoised approximate wavelet coefficients at level 1 .

5. Calculate the value of $\sigma_{1}$ of the wavelet coefficients at level 1 from the difference between the denoised wavelet coefficient at level 1 and the noisy approximate coefficient at the same level.

6. Using $\sigma_{1}$ to denoise the detail wavelet coefficients at level 1 .

7. The last approximate and detail coefficients are combined together to get the denoised signal.

In the simulation test, the AWGN channel is simulated by adding a Gaussian noise to the transmitted signal without affecting the signal synchronization. The simulation will be done by varying the Signal-to-Noise Ratio (SNR) from 5 to $-10 \mathrm{dBs}$. The Peak Signal to Noise Ratio (PSNR) is used to measure the received image quality, and the MSE for the audio signal evaluation.Fig.22shows the PSNR against SNR for Monalisa color image, and Fig.23 shows the MSE against SNR for the input audio signal.

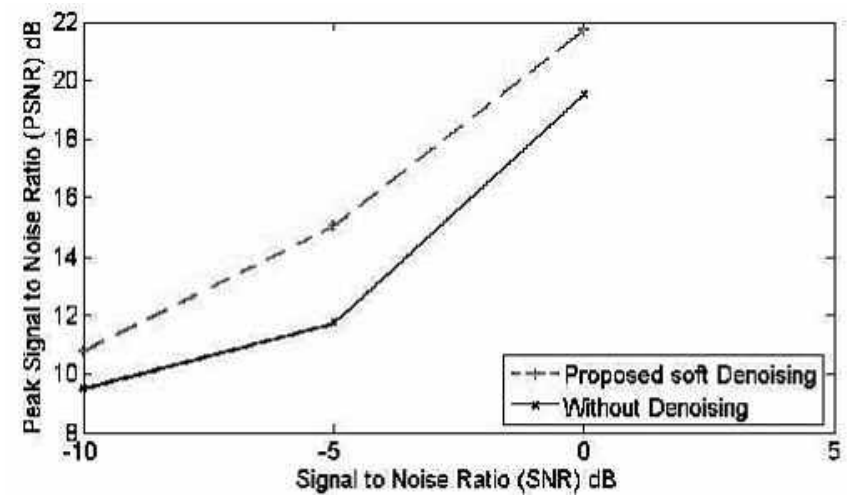

Fig. 22. PSNR against SNR for Monalisa color image signal

A comparison between the CP-OFDM and the proposed system performance for the denoising wavelet techniques shows that a little improvement in PSNR is achieved due to the short length of the modified OFDM symbol relative to the length of the CP-OFDM symbol. Fig. 24shows the perceptual quality of the transmitted image and the received image with $\mathrm{SNR}=0 \mathrm{~dB}$.

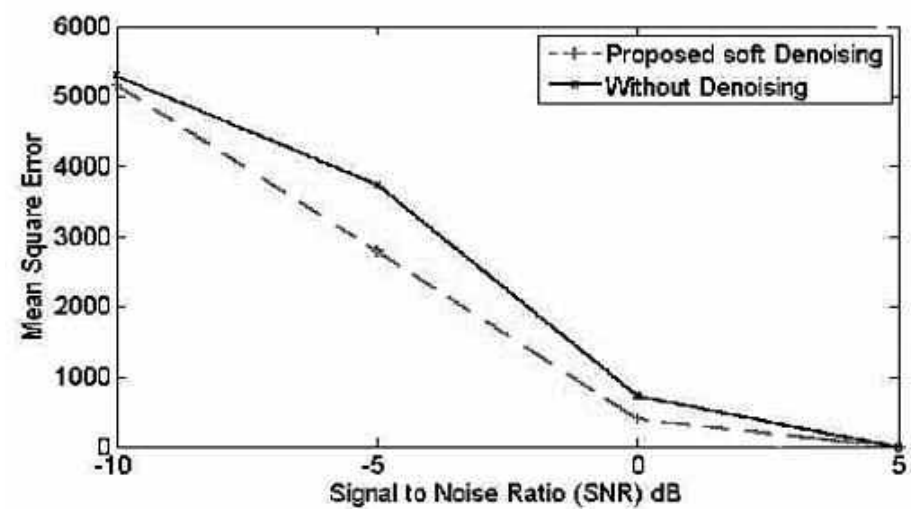

Fig. 23. MSE against SNR for audio signal 
To demonstrate the performance of the proposed denoising system, the comparison between the proposed system and the OFDM-DWT proposed in (M \& Dr.B.V.Uma, 2013) is introduced. The OFDM-DWT system (M \& Dr.B.V.Uma, 2013) uses the Inverse Discrete Wavelet Transform (IDWT)/Discrete Wavelet Transform (DWT) instead of IFFT/FFT in the OFDM system. A generated PN sequences is used in the comparison to test the two systems performance. Table.1 illustrates the performance of the proposed approach relative to the OFDM-DWT.
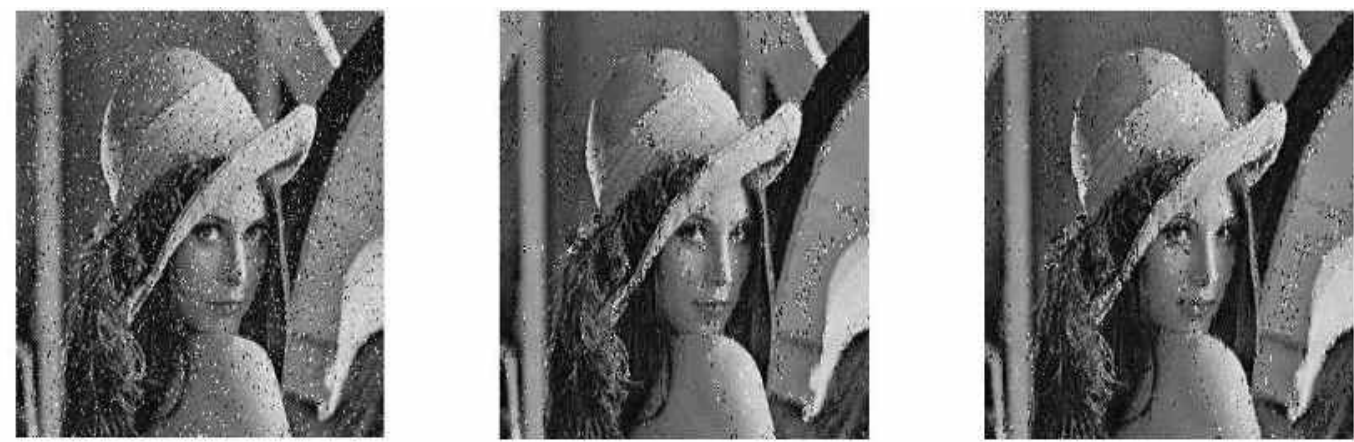

Fig. 24. A Performance comparison between the proposed system and the CPOFDM after using soft denoising when $\mathrm{SNR}=0 \mathrm{db}(\mathrm{a})$ received image without denoising PSNR=19 .3617 (b) received image with the proposed system $\mathrm{PSNR}=21.7884$ (c) received image with the CP-OFDM denoising PSNR=21.3 .56

It's clear that the proposed system outperforms the performance of the OFDM-DWT. The comparison is used the absolute difference between the denoisy and the transmitted signal with the channel noise of $\mathrm{SNR}=22 \mathrm{db}$.

\subsection{Performance analysis of the proposed system withthe AWGN and the fading channel together}

In this Section, the proposed system is tested with the combination of Rician and AWGN channel. To evaluate the performance of the system, Lena image is used as a test signal. The fading criteria will be varied from $0 \%$ to $25 \%$ with the OFDM frame length of 2048.The SNR of the AWGN channel is varied from 5 to $-5 \mathrm{~dB}$. Fig. 25 shows the received image without denoising, and with the denoising technique when $1 \%$ fading delay and $\mathrm{SNR}=5 \mathrm{db}$ Gaussian noise are added. The same result is shown in Fig. 26when 25\% fading and $\mathrm{SNR}=0 \mathrm{db}$ is used. Table. 2 shows the SNR for the received signal at the input of the receiver after the transmission over the Rician fading channel, the SNR of the received signal after the transmission over the AWGN, the SNR of the denoised signal, and the PSNR of the received image. 
Table 1.

Difference between denoised signal and the Transmitted signal for $\mathrm{SNR}=22 \mathrm{~dB}$ for both OFDM-DWT and proposed systems

\begin{tabular}{|c|c|c|c|c|c|c|c|}
\hline \multirow{3}{*}{ 4-Bit } & \multirow{3}{*}{$\begin{array}{c}\text { Transsignal } \\
\text { const. }\end{array}$} & \multicolumn{3}{|c|}{ OFDM-DWT } & \multicolumn{3}{|c|}{ Proposed Denoising } \\
\hline & & \multirow{2}{*}{$\begin{array}{c}\text { Received } \\
\text { signal } \\
\text { constellation } \\
\text { after } \\
\text { Denoising }\end{array}$} & \multicolumn{2}{|c|}{ Difference } & \multirow{2}{*}{$\begin{array}{c}\text { Received } \\
\text { signal } \\
\text { constellation } \\
\text { after } \\
\text { Denoising }\end{array}$} & \multicolumn{2}{|c|}{ Difference } \\
\hline & & & Real & Imag. & & Real & Imag. \\
\hline 0000 & $-3+3 i$ & $\begin{array}{c}-3.0272 \\
+2.8808 \mathrm{i} \\
\end{array}$ & 0.0272 & 0.1192 & $\begin{array}{c}-2.8105+ \\
2.8263 \mathrm{i} \\
\end{array}$ & 0.1895 & 0.1737 \\
\hline 0001 & $-1+3 \mathrm{i}$ & $\begin{array}{c}-1.0804+ \\
2.9525 \mathrm{i}\end{array}$ & 0.0804 & 0.0475 & $\begin{array}{c}-0.8872+ \\
2.8946 \mathrm{i}\end{array}$ & 0.1128 & 0.1054 \\
\hline 0010 & $3+3 i$ & $\begin{array}{c}3.1469+ \\
2.9503 \mathrm{i} \\
\end{array}$ & 0.1469 & 0.0497 & $\begin{array}{c}2.8215+ \\
2.8712 \mathrm{i} \\
\end{array}$ & 0.1785 & 0.1288 \\
\hline 0011 & $1+3 i$ & $\begin{array}{c}0.8712+ \\
3.1746 \mathrm{i}\end{array}$ & 0.1208 & 0.1746 & $\begin{array}{c}0.9457+ \\
2.7797 \mathrm{i}\end{array}$ & 0.0543 & 0.2203 \\
\hline 0100 & $-3-3 i$ & $\begin{array}{c}-2.9841- \\
2.9337 \mathrm{i}\end{array}$ & 0.1592 & 0.0663 & $\begin{array}{c}-2.8156- \\
2.7247 \mathrm{i}\end{array}$ & 0.1844 & 0.2753 \\
\hline 0101 & $-1-3 i$ & $\begin{array}{c}-0.9514- \\
3.0205 \mathrm{i}\end{array}$ & 0.0486 & 0.0205 & $\begin{array}{c}-0.8954- \\
2.9330 \mathrm{i}\end{array}$ & 0.1046 & 0.0670 \\
\hline 0110 & $3-3 i$ & $\begin{array}{l}3.0258- \\
3.0272 \mathrm{i}\end{array}$ & 0.0258 & 0.0272 & $\begin{array}{c}2.7976- \\
2.8537 \mathrm{i} \\
\end{array}$ & 0.2024 & 0.1463 \\
\hline 0111 & $1-3 \mathrm{i}$ & $\begin{array}{l}0.5585 \text { - } \\
2.8909 \mathrm{i}\end{array}$ & 0.4415 & 0.1091 & $\begin{array}{c}0.9972- \\
2.8201 \mathrm{i}\end{array}$ & 0.0028 & 0.1799 \\
\hline
\end{tabular}

Table. 2 proves that the SNR improvement by $30 \mathrm{dBs}$ with the proposed approach relative to the CP-OFDM system is achieved.
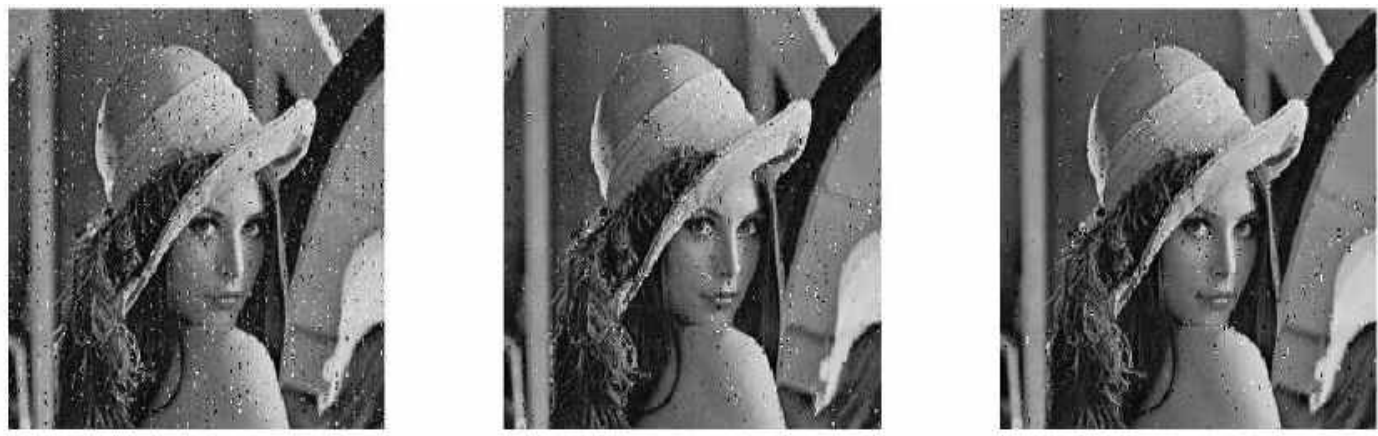

Fig. 25. CP-OFDM, and the proposed system when $1 \%$ fading delay and $\mathrm{SNR}=5 \mathrm{db}$ Gaussian noise is added (a) received image without denoising $\mathrm{PSNR}=21.7663$ (b) received image with the CP-OFDM denoising $\mathrm{PSNR}=24.3318$ (c) received image with the proposed system PSNR=25.4752 

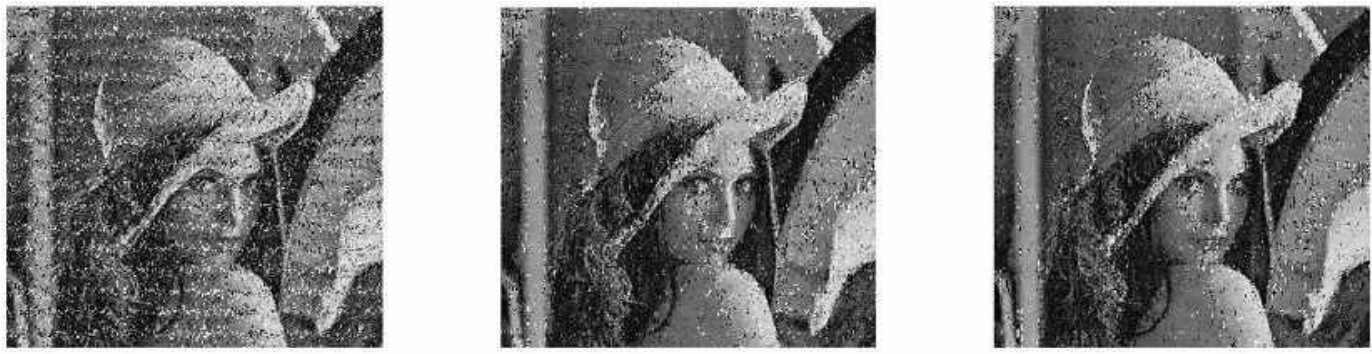

Fig. 26. CP-OFDM, and ZI-OFDM with denoising when $25 \%$ fading delay and $\mathrm{SNR}=0 \mathrm{db}$ Gaussian noise(a) received image without denoising $\mathrm{PSNR}=14.8022$ (b) received image with the CP-OFDM denoising PSNR=17.7931 (c) received image with the proposed system PSNR=18.2096

Table 2.

The performance of the proposed system with the fading and the AWGN channels

\begin{tabular}{|c|c|c|c|c|c|c|}
\hline \multirow[b]{2}{*}{$\begin{array}{c}\text { Fading } \\
\text { delay } \\
(\%)\end{array}$} & \multicolumn{2}{|c|}{ SNR (dBs) after } & \multicolumn{3}{|c|}{ PSNR (dBs) } & \multirow{2}{*}{$\begin{array}{c}\text { SNR } \\
\text { (dBs) after } \\
\text { denoising }\end{array}$} \\
\hline & Fading & AWGN & $\begin{array}{l}\text { Without } \\
\text { denoising }\end{array}$ & $\begin{array}{c}\text { CP-OFDM } \\
\text { with } \\
\text { denoising }\end{array}$ & $\begin{array}{c}\text { ZI-OFDM } \\
\text { with denoising }\end{array}$ & \\
\hline \multirow{3}{*}{1} & \multirow{3}{*}{-6.22} & -7.66 & 21.76 & 24.33 & 25.48 & 13.32 \\
\hline & & -9.72 & 14.62 & 17.633 & 18.48 & 6.91 \\
\hline & & -13.15 & 10.95 & 12.95 & 13.33 & 2.81 \\
\hline \multirow{3}{*}{12.5} & \multirow{3}{*}{-24.11} & -25.31 & 22.75 & 22.91 & 23.22 & 10.87 \\
\hline & & -27.13 & 15.016 & 18.1 & 18.37 & 6.69 \\
\hline & & -30.32 & 10.92 & 13.02 & 13.3 & 2.84 \\
\hline \multirow{3}{*}{25} & \multirow{3}{*}{-27.11} & -28.31 & 22.4 & 22.88 & 22.9 & 10.95 \\
\hline & & -30.12 & 14.8 & 17.8 & 18.21 & 6.84 \\
\hline & & -33.31 & 10.92 & 12.84 & 13.15 & 2.59 \\
\hline
\end{tabular}

In general, the performance of the denoising algorithm is measured using quantitative performance measures such as peak signal-to-noise ratio (PSNR), signal-to-noise ratio (SNR) as well as in terms of visual quality in the case of the images. An ideal denoising procedure requires a priori knowledge of the noise, whereas a practical procedure may not have the required information about the variance of the noise or the noise model. Thus, most of the algorithms assume known variance of the noise and the noise model to compare the performance with different algorithms. It is obvious from the results that, the proposed approach works well in both AWGN channel and fading channels even if the channel characteristic is unknown.

\section{Conclusions}

It is proved that the complexity in the OFDM system can be reduced by replacing the $\mathrm{CP}$ by zero insertion (ZI) before the IFFT process on the OFDM transmitter. Moreover, a signal denoising approach is suggested to be added in the receiver of the OFCM system to reduce the effect of the AWGN channel. The performance analysis with different types of 
data and channels proved that the performance of the proposed system outperforms the performance of the CP-OFDM system. Random, speech, and images signals are used as an input signal for the system to test the performance. The proposed modification approach provides a reduction in the data redundancy by approximately $20 \%$ of the original redundancy with the CP-OFDM system in the fading environmental. Performance of denoising algorithms for the AWGN channel is measured using quantitative performance measures such as peak signal-to-noise ratio (PSNR), signal-to-noise ratio (SNR) as well as in terms of visual quality in the case of the images. The simulation results of the proposed scheme indicate that the proposed system provides a signal enhanced with about 30dbs, according to the SNR for the received signal.

\section{REFERENCES}

[1] Bingham J., "Multicarrier modulation for data transmission, an idea whose time has come," Communications Magazine, IEEE, vol. 28, no. 5, pp. 5-14, May 1990.

[2] Ramjee Prasad, Universal Wireless Personal Communications, 1st ed. Norwood: MA: Artech House, 1998.

[3] Richard van Nee and Ramjee Prasad, OFDM for Wireless Multimedia Communications. Norwood: MA: Artech House, 2000.

[4] B. Noble, I. N. Sneddon, and G. Eason, "On certain integrals of Lipschitz-Hankel type involving products of Bessel functions," Philosophical Transactions of the Royal Society of London, vol. 247, no. 935, pp. 529-551, April 1955.

[5] Z. Wang, G. B. Giannakis, M. de Courville and P. Duhamel B. Muquet, "Cyclic prefixing or zero pading for wireless multicarrier transmissions?," IEEE Transactions on Communications, vol. 50, no. 12, pp. 2136-2148, Dec. 2002.

[6] Ronald N. Bracewell, The Fourier Transform and Its Applications, 3rd ed. California: McGraw-Hill, 2000.

[7] D.L. Donoho and I.M. Johnstone, "Threshold selection for wavelet shrinkage of noisy data," Engineering in Medicine and Biology Society, 1994. Engineering Advances: New Opportunities for Biomedical Engineers. Proceedings of the 16th Annual International Conference of the IEEE, vol. 1, pp. A24-A25, 1994.

[8] Lakhwinder Kaur, Savita Gupta, and R.C. Chauhan, "Image Denoising using Wavelet Thresholding," Dept. of CSE, SLIET, Longowal, pp. 1-4, 2012.

[9] Madhavi Latha and B.Chinna Rao M., "Reconfigurable Wavelet Thresholding for Image Denoising," IJCSNS International Journal of Computer Science and Network Security, vol. 11, no. 3, pp. 222-226, March 2011.

[10] Ken-Hao Liu, Wei-Guang Teng, and Ming-Syan Chen, "Dynamic Wavelet Synopses Management over Sliding Windows in Sensor Networks," IEEE Transactions on Knowledge and Data Engineering, vol. 22, no. 2, pp. 193 - 206, Feb. 2010.

[11] Jianbo Gao, H. Sultan, Jing Hu, and Tung Wen-wen, "Denoising Nonlinear Time Series by Adaptive Filtering and Wavelet Shrinkage: A Comparison," IEEE Signal Processing Letters, vol. 17, no. 3, pp. 237 - 240, March 2010.

[12] II Ryeol Kim and K.E. Barner, "Wavelet Domain Partition- Based Image Denoising," IEEE International Conference on Acoustics, Speech, and Signal Processing, (ICASSP '05), vol. 2, pp. 33 - 36, March 2005.

[13] Govinda Raju. M and Dr.B.V.Uma, "Design and Simulation of Wavelet OFDM with Wavelet Denoising on AWGN Channel," International Journal of Advanced Research in Computer and Communication Engineering, vol. 2, no. 8, pp. 3015-3018, August 2013.

[14] M. Moeneclaey H. , Steendam, "Analysis and Optimization of the Performance of OFDM on Frequency-Selective Time-Selective Fading Channels," IEEE Transactions on Communications, vol. 47, no. 12, pp. 1811-1819, Dec. 1999. 


\section{اتجاهات جديدة لتحسين أداء نظام التقسيم المتعدد للترددات المتعامده}

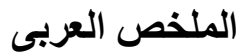

يعتبر نظام التقسيم المتعدد للترددات المتعامدة (OFDM) هو احد نظم الاتصالات الات متعددة الترددات

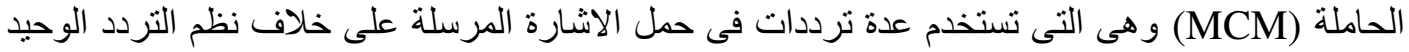

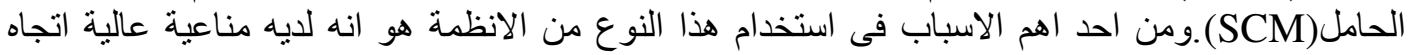

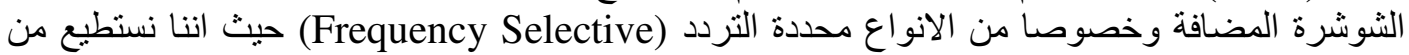

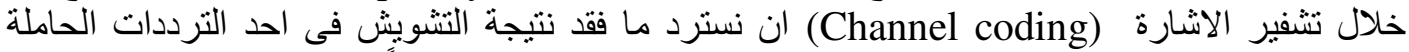

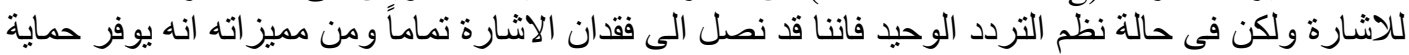

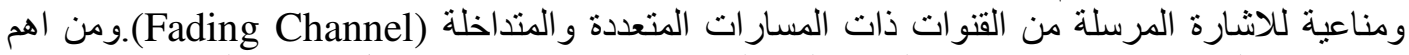

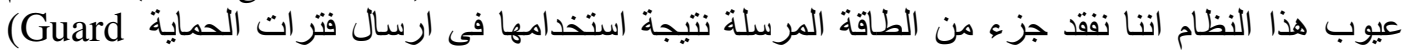

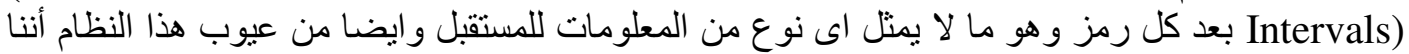

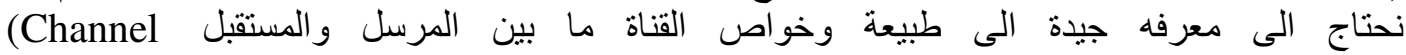

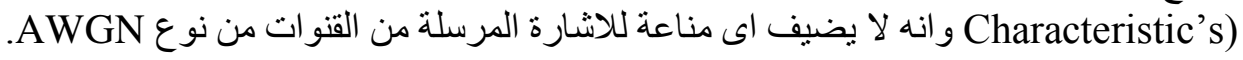

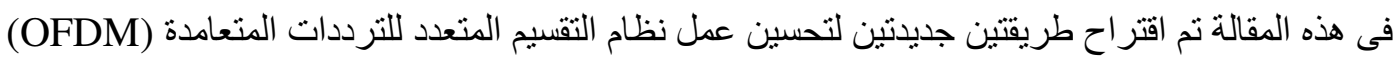

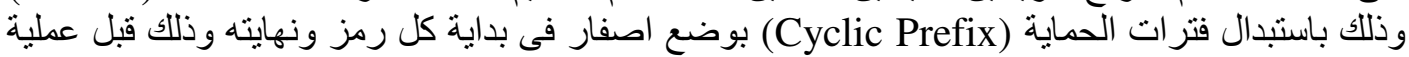

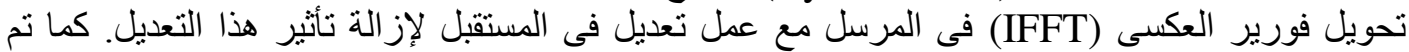

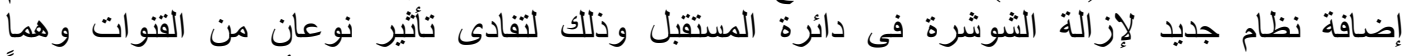

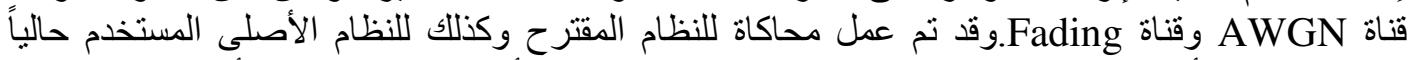

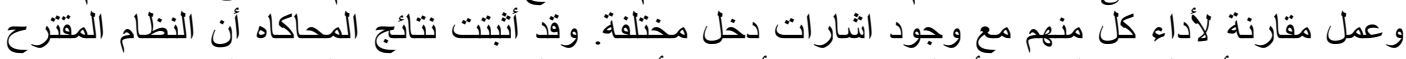

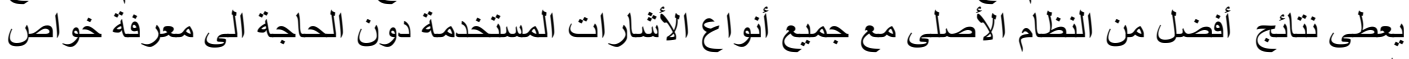

